\title{
Chemical abundances of late-type pre-main sequence stars in the $\sigma$ Orionis cluster
}

\author{
J. I. González Hernández ${ }^{1,2}$, J. A. Caballero ${ }^{3,4}$, R. Rebolo ${ }^{4,5}$, V. J. S. Béjar ${ }^{4}$, \\ D. Barrado y Navascués ${ }^{6}$, E. L. Martín ${ }^{4}$, and M. R. Zapatero Osorio ${ }^{4}$
}

\author{
1 Observatoire de Paris-Meudon, GEPI, 5 place Jules Janssen, 92195 Meudon Cedex, France \\ e-mail: Jonay.Gonzalez-Hernandez@obspm.fr \\ 2 Cosmological Impact of the First STars (CIFIST) Marie Curie Excellence Team, France \\ 3 Dpto. de Astrofísica y Ciencias de la Atmósfera, Facultad de Física, Universidad Complutense de Madrid, 28040 Madrid, Spain \\ 4 Instituto de Astrofísica de Canarias, 38205 La Laguna, Tenerife, Spain \\ 5 Consejo Superior de Investigaciones Científicas, Spain \\ ${ }^{6}$ Laboratorio de Astrofísica Espacial y Física Fundamental, INTA, PO Box 78, 28691 Villanueva de la Cañada, Madrid, Spain
}

Received 16 June 2008 / Accepted 23 August 2008

\section{ABSTRACT}

\begin{abstract}
Context. The young $\sigma$ Orionis cluster is an important location for studying the formation and evolution of stars, brown dwarfs, and planetary-mass objects. Its metallicity, although it is a fundamental parameter, has not been well determined yet.

Aims. We present the first determination of the metallicity of nine young late-type stars in $\sigma$ Orionis.

Methods. Using the optical and near-infrared broadband photometry available in the literature we derive the effective temperatures for these nine cluster stars, which lie in the interval $4300-6500 \mathrm{~K}\left(1-3 M_{\odot}\right)$. These parameters are employed to compute a grid of synthetic spectra based on the code MOOG and Kurucz model atmospheres. We employ a $\chi^{2}$-minimization procedure to derive the stellar surface gravity and atmospheric abundances of $\mathrm{Al}, \mathrm{Ca}, \mathrm{Si}, \mathrm{Fe}, \mathrm{Ni}$ and $\mathrm{Li}$, using multi-object optical spectroscopy taken with WYFFOS+AF2 at the William Herschel Telescope $(\lambda / \delta \lambda \sim 7500)$.

Results. The average metallicity of the $\sigma$ Orionis cluster is $[\mathrm{Fe} / \mathrm{H}]=-0.02 \pm 0.09 \pm 0.13$ (random and systematic errors). The abundances of the other elements, except lithium, seem to be consistent with solar values. Lithium abundances are in agreement with the "cosmic" ${ }^{7} \mathrm{Li}$ abundance, except for two stars which show a $\log \epsilon(\mathrm{Li})$ in the range 3.6-3.7 (although almost consistent within the error bars). There are also other two stars with $\log \epsilon(\mathrm{Li}) \sim 2.75$. We derived an average radial velocity of the $\sigma$ Orionis cluster of $28 \pm 4 \mathrm{~km} \mathrm{~s}^{-1}$.

Conclusions. The $\sigma$ Orionis metallicity is roughly solar.
\end{abstract}

Key words. stars: abundances - stars: pre-main sequence - Galaxy: open clusters and associations: individual: $\sigma$ Orionis

\section{Introduction}

The $\sigma$ Orionis cluster is a nearby, very young region useful for searching for and characterizing substellar objects. The age has been estimated at $3 \pm 2 \mathrm{Ma}$ (Oliveira et al. 2002; Zapatero Osorio et al. 2002a; Sherry et al. 2004) and its heliocentric distance is roughly $360_{-60}^{+70} \mathrm{pc}$ (Brown et al. 1994). The cluster is relatively free of extinction $\left(A_{\mathrm{V}} \ll 1 \mathrm{mag}-\right.$ Lee 1968 ; Béjar et al. 2004) and has a moderate spatial member density (Caballero 2008a) and a high frequency of intermediate-mass stars with discs. A compilation of different determinations of the age, distance, and disc frequency at different mass intervals is provided in Caballero (2007). The cluster contains several dozen brown dwarfs with spectroscopic features of youth and with discs (Béjar et al. 1999; Zapatero Osorio et al. 2002a; Barrado y Navascués et al. 2002; Muzerolle et al. 2003; Kenyon et al. 2005; Burningham et al. 2005; Caballero et al. 2006, 2007). It is also the star forming region with the largest number of candidate isolated planetary-mass objects with followup spectroscopy (Zapatero Osorio et al. 2000, 2002b,c; Martín et al. 2001; Barrado y Navascués et al. 2001; Martín \& Zapatero Osorio 2003). Some of these planetary-mass objects have discs (Zapatero Osorio et al. 2007; Scholz \& Eislöffel 2008; Luhman et al. 2008)
A metallicity detemination in the $\sigma$ Orionis cluster is becoming essential. The derived distance and age of the cluster (from fits to theoretical isochrones) depend on its metallicity, as recently shown by Sherry et al. (2008). At the age of the cluster $(\tau \sim 3 \mathrm{Ma})$, the protoplanetary discs transit from optically thick to optically thin. Giant planets may form at that time (Lissauer 1993; Pollack et al. 1996; Boss 1997; Ida \& Lin 2004). Therefore, the metallicity could play an important rôle in planet formation in $\sigma$ Orionis (e.g. through the planet-metallicity correlation - Fischer \& Valenti 2005).

Cunha et al. (1998) reported that the metallicity of the Orion OB1 association as a whole is $[\mathrm{Fe} / \mathrm{H}]=-0.16 \pm$ $0.11^{1}$, which is similar to other determinations using different samples of stars (e.g., Cunha \& Lambert 1994); see a review of abundance ratios and Galactic chemical evolution in McWilliam (1997). However, there have been very few metallicity studies in the $\sigma$ Orionis cluster. According to Cunha et al. (2000) and references therein, the iron abundance of the early-G star HD 294297 (SO000041, Mayrit 1659068) is slightly subsolar $\left([\mathrm{Fe} / \mathrm{H}]_{\mathrm{HD}} 294297=-0.18\right)$. Nonetheless, although the star is young $\left(\log \epsilon(\mathrm{Li})=2.56^{2}\right)$ and follows the

\footnotetext{
${ }^{1}[\mathrm{Fe} / \mathrm{H}]=\log [N(\mathrm{Fe}) / N(\mathrm{H})]-\log [N(\mathrm{Fe}) / N(\mathrm{H})]_{\odot}$.

${ }^{2} \log \epsilon(\mathrm{Li})=\log [N(\mathrm{Li}) / N(\mathrm{H})]+12$.
} 
spectrophotometric sequence of $\sigma$ Orionis, its cluster membership is in doubt because of its abnormally high proper motion (Caballero 2007). Another star whose metallicity has been investigated with optical spectroscopy is the K3 spectroscopic binary OriNTT 429 AB (Mayrit 1415279 AB). Lee et al. (1994) determined $[\mathrm{Fe} / \mathrm{H}]=-0.43$ and $-0.24 \pm 0.10$ for the primary and the secondary, respectively. Such a difference of iron abundances is questionable if none of the components of the coeval system experiences chemical evolution (e.g. departure from the main sequence). Caballero (2006) measured an average value of $[\mathrm{Fe} / \mathrm{H}]=0.0 \pm 0.1$ from an ensemble of mid-resolution spectra centred on $\mathrm{H} \alpha \lambda 6562.8 \AA$. Using X-ray spectral energy distributions, coronal abundances of cluster stars have also been found to be roughly solar (Skinner et al. 2008) or slightly lower (Sanz-Forcada et al. 2004; Franciosini et al. 2006; López-Santiago \& Caballero 2008).

In this paper, we determine photospheric abundances of iron, lithium and other elements in nine pre-main sequence, late-type stars of $\sigma$ Orionis, with the aim of providing an average, accurate value of its metallicity. Our study improves the $[\mathrm{Fe} / \mathrm{H}]$ estimation presented in Caballero (2006), from where we have taken the original optical spectra.

\section{Observations and data compilation}

Caballero (2006) presented the spectra of 143 stars in a wide area centred on $\sigma$ Ori $\mathrm{AB}$ (the $\mathrm{OB}$ multiple star system that gives the name to the cluster). Multi-object spectra were taken with the Wide Field Fibre Optical Spectrograph (fiber diameter $\sim 1.6 \operatorname{arcsec}$ ) and the robot positioner AutoFib2 (WYFFOS+AF2) at the Observatorio del Roque de los Muchachos, using the $4.2 \mathrm{~m}$ William Herschel Telescope (WHT). We carried out the observations on 2003 Nov. 30, covering the spectral region $\lambda \lambda 6400-6800 \AA$ at an effective resolving power $\lambda / \delta \lambda \sim 7500$ (or $0.83 \AA /$ pixel). The spectra were reduced, wavelength calibrated, and combined in a standard manner using IRAF and the package dohydra. Some preliminary results, like the frequency of accretors, were advanced by Caballero (2005). Further details on the observations, reduction, and analysis of the data and discussion of the results will be presented in a forthcoming paper (Caballero et al., in prep. I)

We chose 25 stars from Caballero (2006) to investigate in detail the metallicity in $\sigma$ Orionis. Their spectra have the highest signal-to-noise ratio of the WYFFOS+AF2 sample and strong Li I $\lambda 6707.8 \AA$ lines in absorption, which identifies them as very young stars. Furthermore, some of these stars have $\mathrm{H} \alpha \lambda 6562.8 \AA$ in strong, asymmetric, and/or broad emission, which is indicative of accretion from a disc. In the full WYFFOS+AF2 sample, there exist stars with higher signal-tonoise ratios, but they are too warm for our purposes (e.g. B2Vp $\sigma$ Ori E [Mayrit 42062]) or are bright GKM dwarfs in the foreground (without any trace of lithium; e.g. G0V HD 294269).

The determination of the optical magnitudes of Mayrit 958292 was affected by its close angular separation to the brighter star TYC 4771-962-1 (Mayrit 968292). Also, the optical spectrum of Mayrit 958292 suffered from an incorrect wavelength calibration in Caballero (2006). As a result, we discarded the star from the input sample of pre-selected stars suitable for our chemical analysis.

The identification number in Caballero (2006), coordinates from the Two Micron All Sky Survey (2MASS - Skrutskie et al. 2006), $V$ magnitude from the All Sky Automated Survey (ASAS-3 - Pojmanski 2002), $i$ magnitude from the Deep Near
Infrared Survey from the Southern Sky (DENIS - Epchtein et al. 1997), and $J, H$, and $K_{\mathrm{s}}$ magnitudes from 2 MASS are given in Table 1 for each of the remaining 24 pre-selected stars. The $V$ magnitudes in the ASAS-3 and Johnson systems are identical. Tabulated $V$ values were computed by averaging all ASAS-3 measurements at less than 15 arcsec to the central 2MASS coordinates of each star and with ASAS-3 photometric quality flags " $\mathrm{A}$ " and "B". The error $\delta V$ is the standard deviation of the mean $V$. Large $\delta V$ values indicate both a low signal-to-noise ratio and an intrinsic photometric variability. For example, the binary SO111112 is one of the most variable stars in the Orion Belt according to Caballero et al. (in prep. II). The $V$ magnitude of SO420742 is an approximate lower limit (Sherry et al. 2004, measured $V \approx 14.72 \mathrm{mag}$ ). Likewise, in Table 2, we provide for each star (when available) its Mayrit designation, Simbad name, IRFM effective temperatures, radial and rotational velocities, and important remarks from the literature and from our spectra. Many of the remarks have been taken from the Mayrit catalogue (Caballero 2008c), although we also provide additional data (e.g. $V_{\mathrm{r}}, v \sin i$, type of $\mathrm{H} \alpha$ emission) mostly from Zapatero Osorio et al. (2002a) and Caballero (2006). See below for details on the computation of the radial and rotational velocities.

\section{Analysis and results}

\subsection{Effective temperatures}

Effective temperatures of the 24 pre-selected stars, $T_{\text {eff }}$, were determined using the infrared flux method (IRFM) and the magnitudes $V, J, H$ and $K_{\mathrm{s}}$ (see Table 2). We used the calibration of the bolometric flux as a function of the colour $V-K_{\mathrm{s}}$ and the magnitude $K_{\mathrm{s}}$ from Alonso et al. $(1995,1999)$, converted into the homogeneous system of Bessell \& Brett (1988). Each of these temperatures was determined by comparing the theoretical fluxes integrated in the 2MASS filters with the observed $J H K_{\mathrm{s}}$ magnitudes. We assumed solar metallicity and surface gravity $\log g=3.9$ for all the stars in the sample. Systematic errors due to a different metallicity (by $+0.1 \mathrm{dex}$ ) and a different surface gravity (by $+0.5 \mathrm{dex}$ ) are in the range $7-100 \mathrm{~K}$ and $2-30 \mathrm{~K}$, respectively, for all stars in the sample. The derived effective temperature was the average of three individual $T_{\text {eff }}$, one for each near-infrared passband, weighted with the inverse of their individual errors, one for each near-infrared passband. To estimate the uncertainties in $T_{\text {eff }}$, we considered a linear transmission of the errors, given that the errors in each photometric magnitude are not totally independent. The error in the average effective temperature accounts for the photometric errors of the observed $J H K_{\mathrm{s}}$ and $V$ magnitudes, the error on the absolute calibration of 2MASS (Cohen et al. 2003), uncertainties on surface gravity $\left(\Delta_{\log g}=0.5 \mathrm{dex}\right)$ and metallicity $\left(\Delta_{[\mathrm{Fe} / \mathrm{H}]}=0.1 \mathrm{dex}\right)$. Further details will be provided by González Hernández \& Bonifacio (in prep.).

A colour excess of $E(B-V)=0.082$ mag towards the $\sigma$ Orionis cluster was derived from the maps of dust infrared emission of Schlegel et al. (1998). This colour excess is consistent with the range of colour excess estimated by Sherry et al. (2008) for all early-type star members of the $\sigma$ Orionis cluster, $0.04 \mathrm{mag}<E(B-V)<0.09 \mathrm{mag}$.

Some of the values might be slightly affected by flux excesses in the near-infrared bands because of circumstellar discs, which make the derived $T_{\text {eff }}$ higher than in young disc-less stars of the same $V$ magnitude. However, we do not see any significant flux excess in our stars except for the Herbig Ae/Be star SO230838 (HD 290772; Gregorio-Hetem \& Hetem 2002). 
Table 1. Identification number in Caballero (2006), 2MASS coordinates, ASAS-3 V, DENIS $i$, and 2MASS $J H K_{\mathrm{s}}$ magnitudes of the 24 preselected stars.

\begin{tabular}{|c|c|c|c|c|c|c|c|}
\hline SO & $\begin{array}{c}\alpha \\
(\mathrm{J} 2000)\end{array}$ & $\begin{array}{c}\delta \\
(\mathrm{J} 2000)\end{array}$ & $\begin{array}{c}V \\
{[\mathrm{mag}]}\end{array}$ & $\begin{array}{c}i \\
{[\mathrm{mag}]}\end{array}$ & $\begin{array}{c}J \\
{[\mathrm{mag}]}\end{array}$ & $\begin{array}{c}H \\
{[\mathrm{mag}]}\end{array}$ & $\begin{array}{c}K_{\mathrm{s}} \\
{[\mathrm{mag}]}\end{array}$ \\
\hline 230838 & 053736.86 & -020817.7 & $9.898 \pm 0.082$ & $9.420 \pm 1.00$ & $8.690 \pm 0.029$ & $8.144 \pm 0.040$ & $7.573 \pm 0.027$ \\
\hline 000041 & 054027.55 & -022543.1 & $10.117 \pm 0.020$ & $9.765 \pm 0.04$ & $9.062 \pm 0.032$ & $8.872 \pm 0.084$ & $8.775 \pm 0.024$ \\
\hline 410305 & 054012.09 & -030528.4 & $11.035 \pm 0.031$ & $10.565 \pm 0.06$ & $9.905 \pm 0.026$ & $9.634 \pm 0.023$ & $9.607 \pm 0.025$ \\
\hline 210734 & 053753.04 & -023334.4 & $11.649 \pm 0.059$ & $10.798 \pm 1.00$ & $9.991 \pm 0.027$ & $9.605 \pm 0.024$ & $9.474 \pm 0.025$ \\
\hline 131161 & 053856.81 & -020454.5 & $12.343 \pm 0.112$ & $11.147 \pm 0.04$ & $10.330 \pm 0.027$ & $9.763 \pm 0.024$ & $9.619 \pm 0.023$ \\
\hline 420039 & 053859.55 & -024508.1 & $12.520 \pm 0.101$ & $11.686 \pm 0.03$ & $11.222 \pm 0.027$ & $10.984 \pm 0.023$ & $10.893 \pm 0.027$ \\
\hline 210434 & 053807.85 & -023131.4 & $12.715 \pm 0.112$ & $11.634 \pm 1.00$ & $10.566 \pm 0.027$ & $9.930 \pm 0.023$ & $9.769 \pm 0.025$ \\
\hline $000005^{a}$ & 053838.23 & -023638.4 & $12.9-14.0$ & $12.340 \pm 0.03$ & $11.158 \pm 0.026$ & $10.465 \pm 0.023$ & $10.312 \pm 0.022$ \\
\hline 211394 & 053715.37 & -023053.4 & $12.986 \pm 0.150$ & $12.176 \pm 1.00$ & $11.364 \pm 0.024$ & $11.006 \pm 0.025$ & $10.877 \pm 0.025$ \\
\hline 420316 & 053854.11 & -024929.8 & $13.164 \pm 0.204$ & $11.729 \pm 0.03$ & $10.829 \pm 0.026$ & $10.310 \pm 0.024$ & $10.126=$ \\
\hline 111112 & 053848.04 & -022714.2 & $13.340 \pm 0.341$ & $11.359 \pm 0.03$ & $10.156 \pm$ & $9.463 \pm 0.026$ & $9.187 \pm 0.019$ \\
\hline 130452 & 053945.15 & -020453.9 & $13.453 \pm 0.215$ & $12.236 \pm 0.04$ & $10.693 \pm$ & $9.960 \pm 0.035$ & $9.556 \pm$ \\
\hline 230062 & 053823.88 & -020542.0 & $13.675 \pm 0.260$ & $12.378 \pm 0.03$ & $11.451 \pm 0.023$ & $10.866 \pm 0.026$ & $10.715 \pm 0.021$ \\
\hline 121137 & 053835.87 & -023043.3 & $13.701 \pm 0.290$ & $12.483 \pm 0.03$ & $11.245 \pm 0.026$ & $10.598 \pm 0.023$ & $10.424 \pm 0.024$ \\
\hline $000006^{a}$ & 053849.17 & -023822.2 & $13.7-15.0$ & $12.891 \pm 0.02$ & $11.389 \pm 0.026$ & $10.663 \pm 0.023$ & $10.511 \pm 0.022$ \\
\hline 430847 & 053932.57 & -023944.0 & $13.756 \pm 0.202$ & $12.385 \pm 0.17$ & $10.820 \pm 0.027$ & $10.104 \pm 0.024$ & $9.917 \pm 0.019$ \\
\hline 430136 & 054022.56 & -023346.9 & $13.951 \pm 0.207$ & $12.473 \pm 0.04$ & $11.158 \pm 0.028$ & $10.533 \pm 0.023$ & $10.362 \pm 0.023$ \\
\hline 420040 & 053827.26 & -024509.7 & $14.192 \pm 0.472$ & $12.847 \pm 0.02$ & $11.955 \pm 0.028$ & $10.792 \pm 0.026$ & $9.944 \pm 0.028$ \\
\hline 440660 & 053954.66 & -024634.1 & $14.253 \pm 0.354$ & $12.757 \pm 0.03$ & $11.054 \pm 0.028$ & $10.251 \pm 0.024$ & $9.832 \pm 0.024$ \\
\hline 121112 & 053840.27 & -023018.5 & $14.273 \pm 0.389$ & $12.839 \pm 0.02$ & $11.512 \pm 0.026$ & $10.763 \pm 0.023$ & $10.395 \pm 0.025$ \\
\hline 420147 & 053852.01 & -024643.7 & $14.301 \pm 0.305$ & $12.719 \pm 0.02$ & $11.518 \pm 0.026$ & $10.774 \pm 0.024$ & $10.421 \pm 0.021$ \\
\hline 430967 & 053925.20 & -023822.0 & $14.344 \pm 0.341$ & $13.028 \pm 0.16$ & $11.307 \pm 0.031$ & $10.451 \pm 0.023$ & $10.002 \pm 0.023$ \\
\hline 140159 & 054005.11 & -021959.1 & $14.493 \pm 0.324$ & $12.950 \pm 0.03$ & $11.459 \pm 0.026$ & $10.767 \pm 0.024$ & $10.542 \pm 0.021$ \\
\hline 420742 & 053839.82 & -025646.2 & $\gtrsim 14.5$ & $12.778 \pm 0.02$ & $11.413 \pm 0.027$ & $10.744 \pm 0.023$ & $10.439 \pm 0.021$ \\
\hline
\end{tabular}

${ }^{a}$ For these stars we provide two values of the $V$ magnitude. The first number corresponds to the ASAS- $3 V$ magnitude and other values from Wolk (1996, SO000005) and Sherry et al. (2004, SO000006).

It is an intense accretor, IRAS source, X-ray emitter, F6-type star much warmer than tabulated in Table 2. For this star, IRFM temperatures derived from the three 2 MASS filters differ by $600 \mathrm{~K}$, while for other stars in the sample the three IRFM temperatures are usually consistent within $100 \mathrm{~K}$. Besides, for another two stars, S0000005 and SO000006, two values of the $V$ magnitude are given, providing two temperature determination (see the discussion in Sect. 3.2).

\subsection{Final sample for chemical analysis}

Several of the 24 pre-selected stars are not appropiate for the chemical analysis. In particular, there are 11 stars that have $T_{\text {eff }} \lesssim 4000 \mathrm{~K}$, for which the IRFM temperature estimates are not reliable because the calibration of the bolometric flux is uncertain at these temperatures. In addition, our spectral fitting procedure is not so accurate at these low temperatures because we do not include molecular bands, in particular $\mathrm{TiO}$ bands, which appear to be required for the spectral fitting at $T_{\text {eff }} \lesssim 4200 \mathrm{~K}$. Among the discarded "cool" stars, one is a close binary resolved with adaptive optics observations (SO111112; $\rho=0.40 \pm 0.08$ arcsec - Caballero 2005) and another one is a spectroscopic triple with a high apparent rotational velocity (SO130452 - Alcalá et al. 2000). Both of them likely harbour discs because they display flux excesses in the near-infrared.

The stars S0000005 and SO000006 are two X-ray emitters with very strong Li I $\lambda 6708 \AA$ lines. The ASAS- $3 V$ magnitudes provide effective temperatures derived from IRFM $\left(T_{\text {eff }}=4935\right.$ and $4450 \mathrm{~K}$, respectively) that must be too high because they produces too large lithium abundances $(\log \epsilon(\mathrm{Li}) \sim 4.4)$. Other $V$ magnitudes tabulated in the literature for both stars
(Zapatero Osorio et al. 2002a; Sherry et al. 2004) give IRFM effective temperatures below $4000 \mathrm{~K}$.

In order to perform a detailed chemical analysis, we discarded the 13 "cool" stars, including S0000005 and SO000006, the very active Herbig Ae/Be star SO230838, and the possible spectroscopic binary SO410305 (Sect. 3.3) and retained only nine stars appropiate for spectral fitting.

According to the Siess et al. (2000) models for very young ages, the derived effective temperatures of the nine selected stars correspond to approximate spectral types in the interval F5 to K6.

\subsection{Radial and rotational velocities}

For all stars in the pre-selected sample (including the nine stars suitable for chemical analysis), we extracted their radial velocities by cross-correlating their WYFFOS + AF2 spectra with the spectrum of a K3V template star taken from the $\mathrm{S}^{4} \mathrm{~N}$ database (HD 160346 [HIP 86400] - Allende Prieto et al. 2004), using the software MOLLY developed by Marsh. We also computed the optimal rotational velocity, $v \sin i$, by subtracting broadened versions of the template star, in steps of $5 \mathrm{~km} \mathrm{~s}^{-1}$, and minimizing the residual. We used a spherical rotational profile with linearized limb-darkening $\epsilon=0.65$ (Al-Naimiy 1978). We took into account the instrumental broadening, which was estimated at about $40 \mathrm{~km} \mathrm{~s}^{-1}$. Radial and rotational velocities are provided in Table 2.

All except one of the stellar radial velocities given in Table 2 are in agreement with the canonical value of the average cluster radial velocity at $V_{\mathrm{r}} \approx+30.9 \pm 0.9 \mathrm{~km} \mathrm{~s}^{-1}$ (Wilson 1953 ; Morrell \& Levato 1991; Zapatero Osorio et al. 2002a; Kenyon et al. 2005; Maxted et al. 2008; Sacco et al. 2008). The outlier, 
Table 2. Names, IRFM effective temperatures, radial and rotational velocities, and remarks from the literature of the 24 pre-selected stars.

\begin{tabular}{|c|c|c|c|c|c|c|}
\hline $\mathrm{SO}^{a}$ & Mayrit $^{b}$ & $\begin{array}{l}\text { Simbad } \\
\text { name }\end{array}$ & $\begin{array}{l}T_{\text {eff }} \\
{[\mathrm{K}]}\end{array}$ & $\begin{array}{c}V_{\mathrm{r}}^{c} \\
{\left[\mathrm{~km} \mathrm{~s}^{-1}\right]}\end{array}$ & $\begin{array}{c}v \sin i \\
{\left[\mathrm{~km} \mathrm{~s}^{-1}\right]}\end{array}$ & Remarks $^{d}$ \\
\hline 230838 & $\ldots$ & HD 290772 & $6000 \pm 600^{e}$ & $+23.3 \pm 0.4$ & $30 \pm 5$ & Herbig Ae/Be, IRAS, $\rho>30 \operatorname{arcmin}$ \\
\hline 000041 & 1659068 & HD 294297 & $6450 \pm 105$ & $+24.6 \pm 0.2$ & $<20$ & {$[\mathrm{Fe} / \mathrm{H}], V_{\mathrm{r}}=+25.0 \mathrm{~km} \mathrm{~s}^{-1}$} \\
\hline 410305 & $\ldots$ & HD 294308 & $6250 \pm 90$ & $-37.2 \pm 0.2$ & $<20$ & $\rho>30 \operatorname{arcmin}$ \\
\hline 210734 & 789281 & 2E 1454 & $5235 \pm 100$ & $+24.0 \pm 0.3$ & $30 \pm 5$ & $\mathrm{H} \alpha$ in broad emission \\
\hline 131161 & $\ldots$ & $\ldots$ & $4765 \pm 115$ & $+23.4 \pm 0.3$ & $<20$ & $\rho>30$ arcmin, in NE nebulosity \\
\hline 420039 & 591158 & [W96] 4771-0026 & $5935 \pm 195$ & $+33.7 \pm 0.3$ & $60 \pm 5$ & {$[\mathrm{~S}$ II $]$ and $[\mathrm{N}$ II $]$ in emission } \\
\hline 210434 & 615296 & 2E 1459 & $4630 \pm 110$ & $+24.5 \pm 0.4$ & $<20$ & $\mathrm{H} \alpha$ in faint emission \\
\hline 000005 & 105249 & [W96] rJ053838-0236 & $4935-4000^{f}$ & $+25.1 \pm 0.3$ & $<20$ & $\mathrm{H} \alpha$ in faint emission, $V_{\mathrm{r}}=+42 \pm 10 \mathrm{~km} \mathrm{~s}^{-1}$ \\
\hline 211394 & 1374283 & Mayrit 1374283 & $5300 \pm 210$ & $+27.8 \pm 0.3$ & $<20$ & high background around $\mathrm{H} \alpha$ \\
\hline 420316 & 822170 & RX J0538.9-0249 & $4520 \pm 175$ & $+31.8 \pm 0.4$ & $<20$ & $\mathrm{H} \alpha$ in faint emission, $v \sin i=28 \mathrm{~km} \mathrm{~s}^{-1}$ \\
\hline 111112 & $528005 \mathrm{AB}$ & [W96] 4771-899 AB & $\$ 3500$ & $+30.7 \pm 0.4$ & $<20$ & $\begin{array}{l}\text { classical T Tau, resolved binary, } \\
V_{\mathrm{r}}=+31 \pm 10 \mathrm{~km} \mathrm{~s}^{-1}\end{array}$ \\
\hline 130452 & $\cdots$ & RX J0539.8-0205 ABC & $\$ 3800$ & $+20.0 \pm 0.5$ & $60 \pm 5$ & $\begin{array}{l}\mathrm{H} \alpha \text { in broad asymmetric emission, } \\
\text { spectroscopic triple, } \rho>30 \text { arcmin, } \\
\text { in NE nebulosity, }[\mathrm{S} \mathrm{II}] \text { and }[\mathrm{N} \mathrm{II}] \text { in emission, } \\
V_{\mathrm{r}}=+27.8 \pm 0.9 \mathrm{~km} \mathrm{~s}^{-1}\end{array}$ \\
\hline 230062 & $\ldots$ & $\ldots$ & $4585 \pm 225$ & $+22.6 \pm 0.3$ & $<20$ & H $\alpha$ in very faint emission, $\rho>30 \operatorname{arcmin}$ \\
\hline 121137 & 344337 & 2E 1468 & $4370 \pm 310$ & $+28.9 \pm 0.4$ & $70 \pm 5$ & $\begin{array}{l}\mathrm{H} \alpha \text { in broad asymmetric emission, } \\
V_{\mathrm{r}}=+35 \pm 7 \mathrm{~km} \mathrm{~s}^{-1}, v \sin i=80 \pm 15 \mathrm{~km} \mathrm{~s}^{-1}\end{array}$ \\
\hline 000006 & 157155 & [W96] rJ053849-0238 & $4450-3500^{f}$ & $+34.1 \pm 0.7$ & $<20$ & $\mathrm{H} \alpha$ in faint, broad emission \\
\hline 430847 & 750107 & $\ldots$ & $\$ 3800$ & $+30.7 \pm 0.5$ & $<20$ & $\mathrm{H} \alpha$ in faint emission \\
\hline 430136 & 1471085 & Kiso A-0904 105 & $\$ 4000$ & $+28.6 \pm 0.4$ & $60 \pm 5$ & $\begin{array}{l}\mathrm{H} \alpha \text { in faint asymmetric emission, } \\
\text { close to Horsehead, [S } \mathrm{II}] \text { and }[\mathrm{N} \text { II] in emission, }\end{array}$ \\
\hline 420040 & 609206 & V505 Ori & $\$ 3800$ & $+29.5 \pm 0.7$ & $<20$ & classical T Tau, $V_{\mathrm{r}}=+48 \pm 10 \mathrm{~km} \mathrm{~s}^{-1}$ \\
\hline 440660 & 1223121 & V606 Ori & $\$ 3800$ & $+29.3 \pm 0.4$ & $<20$ & $\begin{array}{l}\text { H } \alpha \text { in strong, broad, asymmetric emission, } \\
\text { class II, [N II] in emission }\end{array}$ \\
\hline 121112 & 348349 & Haro 5-13 & $\$ 3800$ & $+34.3 \pm 0.4$ & $<20$ & classical T Tau \\
\hline 420147 & 653170 & RU Ori & $\$ 3800$ & $+32.0 \pm 0.6$ & $<20$ & classical T Tau \\
\hline 430967 & 622103 & BG Ori & $\$ 3500$ & $+27.0 \pm 0.6$ & $<20$ & $\begin{array}{l}\mathrm{H} \alpha \text { in strong, broad, asymmetric emission, } \\
\text { class II, [S II] in emission }\end{array}$ \\
\hline 140159 & 1541051 & [NYS99] C-05 & $\$ 3500$ & $+27.5 \pm 0.4$ & $<20$ & $\mathrm{H} \alpha$ in faint, broad emission \\
\hline 420742 & 1248183 & [SWW2004] 125 & $\lesssim 3500$ & $+29.3 \pm 0.5$ & $<20$ & $\mathrm{H} \alpha$ in faint emission \\
\hline
\end{tabular}

${ }^{a}$ Identification number in Caballero (2006); ${ }^{b}$ mayrit designation in Caballero (2008c); ${ }^{c}$ radial velocities extracted from cross-correlation with a $\mathrm{K} 3 \mathrm{~V}$ template star (see main text); ${ }^{d}$ important remarks from the literature (see main text for references); ${ }^{e}$ strong X-ray emitter (see Sect. 3.1); ${ }^{f}$ derived $T_{\text {eff }}$ from the two different $V$ magnitudes given in Table 1.

a late F-star with lithium $\left(T_{\text {eff }}=6250 \pm 90 \mathrm{~K}, \quad V_{\mathrm{r}}=\right.$ $-34 \pm 3 \mathrm{~km} \mathrm{~s}^{-1}$; SO410305), could be a spectroscopic binary. Indeed, the star with the second most discordant $V_{\mathrm{r}}$ is the spectroscopic triple $\mathrm{SO} 130452\left(V_{\mathrm{r}}=+20.0 \pm 0.5 \mathrm{~km} \mathrm{~s}^{-1}\right)$.

We have measured an average radial velocity $\overline{V_{\mathrm{r}}}=$ $+27.9 \pm 4.0 \mathrm{~km} \mathrm{~s}^{-1}(N=23)$, where the error is the standard deviation of the mean. Since the average error in the individual measurements of the cross-correlation method is $\overline{\delta V_{\mathrm{r}}}=0.4 \mathrm{~km} \mathrm{~s}^{-1}$, the simplest interpretation is that $\sigma_{V_{\mathrm{r}}} \sim$ $4 \mathrm{~km} \mathrm{~s}^{-1}$ would be the cluster velocity dispersion. However, other authors (e.g. Sacco et al. 2008) have derived lower velocity dispersions, at $\sigma_{V_{\mathrm{r}}} \sim 1 \mathrm{~km} \mathrm{~s}^{-1}$. This value is of fundamental importance for some theoretical scenarios but, unfortunately, has not been well treated in the literature for the $\sigma$ Orionis cluster.

\subsection{Metallicities and gravities}

Using the derived effective temperatures, we tried to infer the surface gravity, $\log g$, and the metallicity, $[\mathrm{Fe} / \mathrm{H}]$, of the nine selected stars, using a minimization routine that compares several features of the stellar spectra with a grid of synthetic spectra (see González Hernández et al. 2004, 2005, 2008). The synthetic spectra were computed using the local thermodynamic equilibrium (LTE) code MOOG (Sneden 1973), a grid of LTE model atmospheres (Kurucz 1993), and atomic line data extracted from the Vienna Atomic Line Database (VALD, Piskunov 1995). The oscillator strengths of relevant lines were adjusted until they reproduced the solar atlas (Kurucz et al. 1984) with solar element abundances (Grevesse et al. 1996). The adopted abundances were: $\log \epsilon(\mathrm{Al})_{\odot}=6.47, \log \epsilon(\mathrm{Si})_{\odot}=7.55, \log \epsilon(\mathrm{Ca})_{\odot}=6.36$, $\log \epsilon(\mathrm{Fe})_{\odot}=7.50$, and $\log \epsilon(\mathrm{Ni})_{\odot}=6.25$. Element abundances (calculated assuming LTE) were computed using the expression $[\mathrm{X} / \mathrm{H}]=\log [N(\mathrm{X}) / N(\mathrm{H})]_{\text {star }}-\log [N(\mathrm{X}) / N(\mathrm{H})]_{\odot}$, where $N(\mathrm{X})$ is the number density of atoms.

We selected 12 spectral features containing in total 31 lines of $\mathrm{Fe} \mathrm{I}$ and, in some cases, six lines of $\mathrm{Ca} \mathrm{I}$, with excitation potentials between 1 and $5 \mathrm{eV}$. For each given iron abundance in the interval $[\mathrm{Fe} / \mathrm{H}]<0$, the calcium abundance was fixed according to the Galactic trend of calcium (Bensby et al. 2005), while for $[\mathrm{Fe} / \mathrm{H}]>0$, we assumed $[\mathrm{Ca} / \mathrm{Fe}]=0$.

Microturbulence, $\xi$, was fixed in each atmospheric model according to the calibration as a function of effective temperature and surface gravity. Such a calibration has been derived for stars of the solar neighbourhood with approximately solar metallicity (Allende Prieto et al. 2004). 

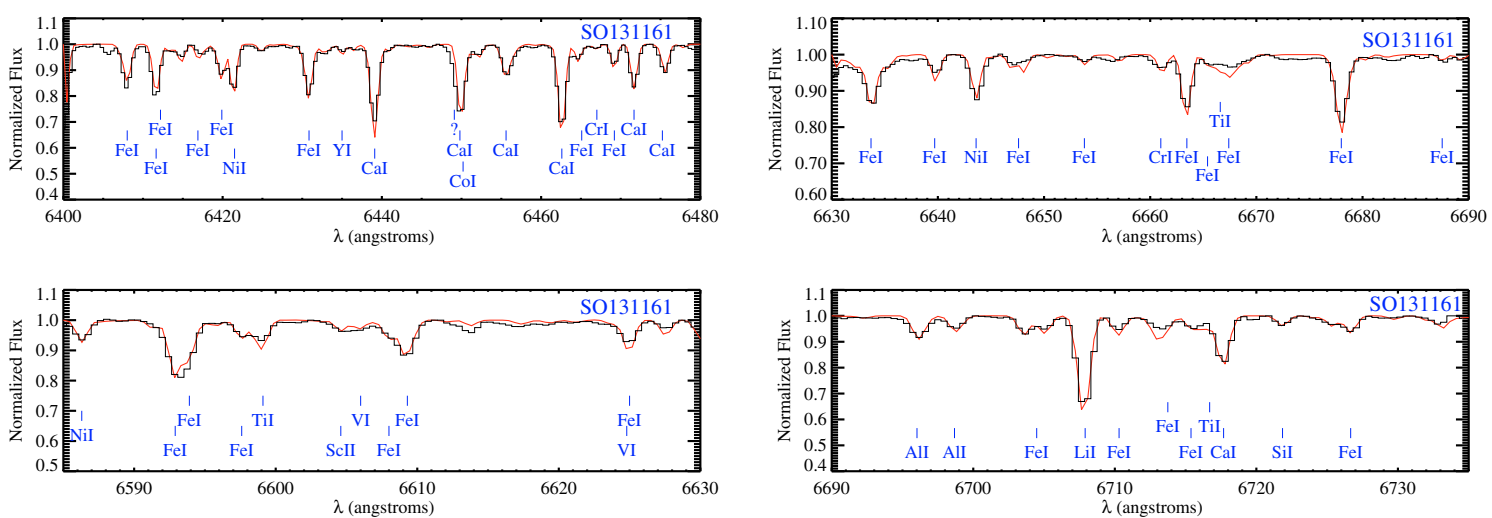

Fig. 1. WYFFOS+AF2 spectrum of SO131161 at the four investigated spectral intervals in comparison with synthetic spectra computed for the element abundances shown in Table 3. Some absorption features are marked.

Table 3. Basic stellar parameters of the nine selected stars.

\begin{tabular}{|c|c|c|c|c|c|c|c|c|c|}
\hline SO & $\begin{array}{l}T_{\text {eff }} \\
{[\mathrm{K}]}\end{array}$ & $\log g$ & $\begin{array}{c}E W(\mathrm{Li} \mathrm{I})^{a} \\
{[\AA]}\end{array}$ & {$[\mathrm{Fe} / \mathrm{H}]$} & {$[\mathrm{Al} / \mathrm{H}]$} & {$[\mathrm{Si} / \mathrm{H}]$} & {$[\mathrm{Ca} / \mathrm{H}]$} & {$[\mathrm{Ni} / \mathrm{H}]$} & $\log \epsilon(\mathrm{Li})_{\mathrm{NLTE}}$ \\
\hline 000041 & 105 & & $04 \pm 0.01$ & 0 & 10 & 13 & -0.12 & -0.0 & \\
\hline 210734 & 0 & & 1 & -0.02 & 1.12 & 3 & 0.03 & -0.1 & \\
\hline 131161 & $65 \pm 115$ & $9 \pm 0.3$ & $0.42 \pm 0.01$ & $0.13 \pm 0.10$ & $0.03 \pm 0.12$ & $0.23=$ & $0.18 \pm$ & $0.18 \pm 0.14$ & 0.18 \\
\hline 420039 & $35 \pm 195$ & $.7 \pm 0.3$ & $\leq 0.14^{b}$ & $-0.10 \pm 0.15$ & $0.15 \pm 0.14$ & $0.00 \pm$ & $-0.22 \pm 0.21$ & $-0.10 \pm 0.13$ & 0.31 \\
\hline 210434 & $30 \pm 110$ & $8 \pm 0.3$ & $0.45 \pm 0.01$ & $0.08 \pm 0.10$ & $-0.04 \pm 0.12$ & $0.23=$ & $0.15 \pm$ & $0.15=$ & 0.18 \\
\hline 211394 & $00 \pm 210$ & $0 \pm 0.3$ & $0.22 \pm 0.01$ & $-0.15 \pm 0.10$ & -0.05 & $0.00=$ & -0.20 & -0.10 & 0.31 \\
\hline 420316 & $20 \pm 175$ & $0 \pm 0.3$ & $0.44 \pm 0.01$ & -0.08 & -0.1 & 0.20 & -0.15 & 0.07 & 3.16 \\
\hline 230062 & $85 \pm 225$ & $4.1 \pm 0.3$ & $0.49 \pm 0.01$ & $0.02 \pm 0.17$ & $-0.10 \pm$ & $0.10=$ & $-0.07=$ & 0.10 & $3.61 \pm$ \\
\hline 121137 & $70 \pm 310$ & $9 \pm 0.3$ & $\leq 0.47^{b}$ & $0.00 \pm$ & & $0.05=$ & -0.20 & $0.07 \pm$ & $3.25 \pm$ \\
\hline Cluster $^{c}$ & $\ldots$ & . & $\ldots$ & $-0.02 \pm 0.09$ & $-0.03 \pm 0.10$ & $0.12 \pm 0.09$ & $-0.07 \pm 0.15$ & $0.02 \pm 0.12$ & \\
\hline
\end{tabular}

${ }^{a}$ Equivalent width of Li I $\lambda 6708 \AA{ }^{b}{ }^{b}$ the lithium line is blended with the Fe I lines at $6703-5 \AA$ due to high rotational velocity of the star; ${ }^{c}$ average abundances of the $\sigma$ Orionis cluster. Uncertainties are computed from standard deviations of the measurements from each star.

We imposed the surface gravity and the metallicity to vary, in steps of $0.05 \mathrm{dex}$, in the intervals $2.50<\log g<4.50$ and $-0.50<[\mathrm{Fe} / \mathrm{H}]<0.50$, respectively. The comparison of this grid with the observed spectra, using a bootstrap Monte Carlo method for each $T_{\text {eff }}$, gives the most likely values of $\log g$ and $[\mathrm{Fe} / \mathrm{H}]$. The values of the trio $T_{\mathrm{eff}}-\log g-[\mathrm{Fe} / \mathrm{H}]$ are provided in Table 3. All the stars show a metallicity in approximate agreement with the solar value within the error bars.

Our spectral-fitting technique also allows us to determine the veiling, defined as $F_{\text {disc }} / F_{\text {star }}$, where $F_{\text {disc }}$ and $F_{\text {star }}$ are the flux contributions of the disc and the continuum of the star, respectively. We have not found any clear evidence of veiling in the analysed spectral region $(\lambda \lambda 6400-6800 \AA)$ of the nine selected stars.

\subsection{Stellar abundances}

Adopting the stellar parameters given in Table 3, we determined the abundances of aluminium, calcium, and nickel from several features of each element. The silicon and lithium abundances were derived from only one spectral feature ${ }^{3}$. In Fig. 1, we display all spectral regions analysed in this work for a representative star of the selected sample. The element abundances are presented in Table 3. The errors on the element abundances show

3 The silicon feature is Si I $\lambda 6721.8 \AA$. their sensitivity to the uncertainties on the effective temperature, $\Delta_{T_{\text {eff }}}$, surface gravity, $\Delta_{\log g}$, and the dispersion of the measurements from different spectral features, $\Delta_{\sigma}$. The errors $\Delta_{\sigma}$ were estimated as $\Delta_{\sigma}=\sigma / N^{1 / 2}$, where $N$ is the number of features analysed of each element and $\sigma$ is the standard deviation of the $N$ measurements. The errors $\Delta_{T_{\text {eff }}}$ and $\Delta_{\log g}$ were determined as $\Delta_{T_{\text {eff }}}=\left(\sum_{i=1}^{N} \Delta_{T_{\text {eff }}, i}\right) / N$ and $\Delta_{\log g}=\left(\sum_{i=1}^{N} \Delta_{T_{\log g}, i}\right) / N$. For silicon and lithium abundances, the error associated with the dispersion of the measurements, $\sigma$, was assumed to be the average dispersion of iron, calcium, and nickel abundances (i.e. $\Delta_{\sigma} \equiv \bar{\sigma}$ ). The total error given in Table 3 was derived using the expresion $\Delta_{\text {tot }}^{2}=\Delta_{\sigma}^{2}+\Delta_{T_{\text {eff }}}^{2}+\Delta_{\log g}^{2}$.

All the element abundances were derived with the assumption of local thermodynamical equilibrium, except for lithium, for which we provided its non-equilibrium (non-LTE) abundance. We estimated the non-LTE abundance correction for this element from the theoretical LTE and non-LTE curves of growth in Pavlenko \& Magazzù (1996).

\section{Discussion}

\subsection{Stellar parameters and heliocentric distance}

We display in Fig. 2 two diagrams involving basic stellar parameters of the selected stars in comparison with several theoretical evolutionary tracks taken from Siess et al. (2000), for several 

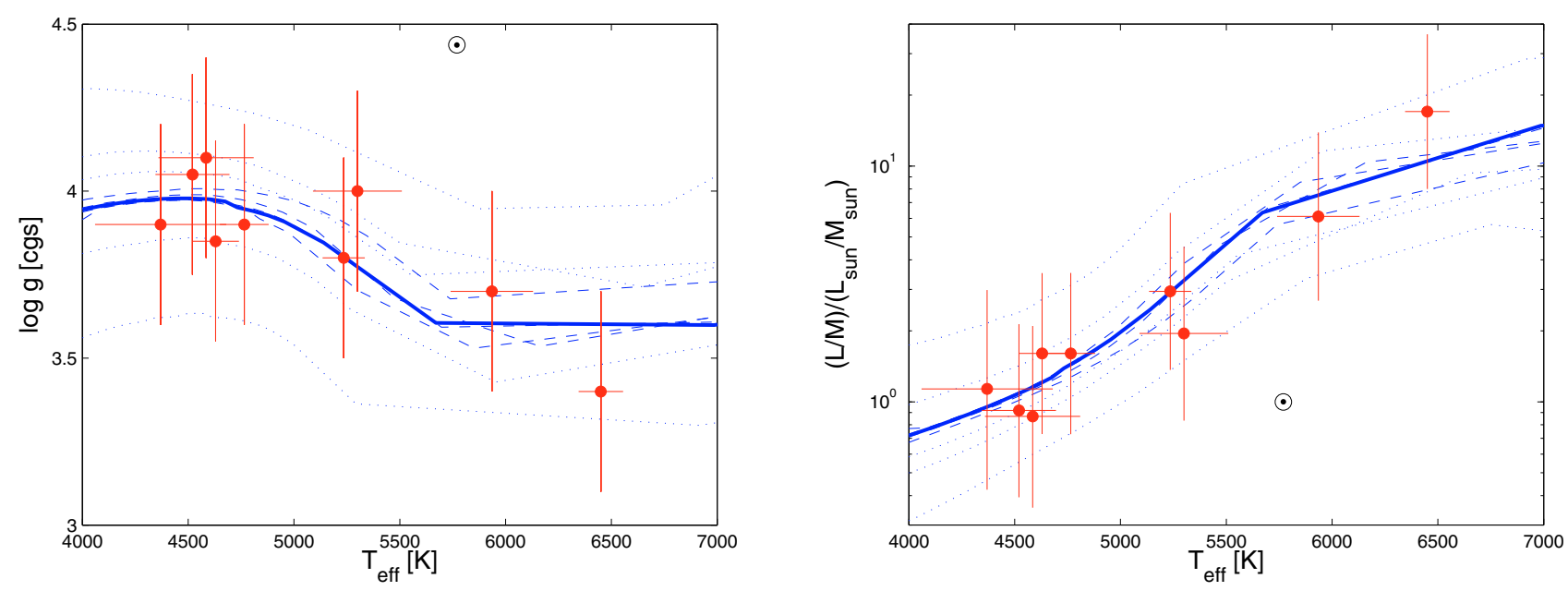

Fig. 2. Gravity $(\log g$, left $)$ and luminosity per unit of mass normalized to the Sun $(\mathcal{L}$, right $)$ as functions of effective temperature $\left(T_{\text {eff }}\right)$ of the nine selected stars (filled circles with error bars). A number of Siess et al. (2000) theoretical evolutionary tracks for different ages, metallicities and overshooting are plotted. Dotted lines: tracks for $Z=0.02$, overshooting, and 10, 5, 4, 2, and $1 \mathrm{Ma}$, from top to bottom; dashed lines: tracks for $3 \mathrm{Ma}$, no overshooting, and $Z=0.01,0.03$, and 0.04 , from top to bottom; and thick solid line: track for $3 \mathrm{Ma}, Z=0.02$, and overshooting. The solar symbol, $\odot$, indicates the position of the Sun.

ages and metallicities. The derived stellar parameters are consistent with almost all the theoretical tracks due to the large error on surface gravity. However, their central values are very well reproduced by the solid line that represents the 3 Ma-old, overshooting track. Ages lower than $2 \mathrm{Ma}$ or larger than $4 \mathrm{Ma}$ are marginally consistent with the stellar parameters of the selected stars. The theoretical tracks are only sensitive to metallicity for effective temperatures higher than $T_{\text {eff }} \sim 4800 \mathrm{~K}$.

In the right panel of Fig. 2, we plot the luminosity per unit of mass, normalized to this quantity in the Sun:

$\mathcal{L}=(L / M) /\left(L_{\odot} / M_{\odot}\right)$.

Using the well known expressions for the bolometric luminosity and the surface gravity, it is derived that:

$L / M=4 \pi G \sigma \frac{T_{\mathrm{eff}}^{4}}{g}$,

and, therefore,

$\mathcal{L}=\frac{g_{\odot}}{g}\left(\frac{T_{\text {eff }}}{T_{\text {eff }, \odot}}\right)^{4}$.

Therefore, $\mathcal{L} \propto g^{-1} T_{\text {eff }}^{4}$ at a given metallicity, which means that the normalized luminosity per unit of mass only depends on the derived stellar parameters.

In addition, in Fig. 3 we display the $\mathcal{L}$ vs. $J$ diagram and several theoretical evolutionary tracks for different heliocentric distances to the $\sigma$ Orionis cluster. In this case, all selected stars, except three, are close to the tracks for distances in the range $350 \mathrm{pc}<d<500 \mathrm{pc}$. These three stars are the hottest stars in the sample (SO000041 $-T_{\text {eff }}=6450 \pm 105 \mathrm{~K}-$, SO420039$T_{\text {eff }}=5935 \pm 195 \mathrm{~K}-$ and SO211394 $-T_{\text {eff }}=5300 \pm 210 \mathrm{~K}-$ ). SO000041 is marginally consistent with the track at $d=400 \mathrm{pc}$ but still too luminous for its expected position in this diagram (see Sect. 4.2). For the other two stars, one should expect $J$ magnitudes in the range $9 \mathrm{mag} \lesssim J \lesssim 10 \mathrm{mag}$, but surprinsingly they have $J \sim 11.3 \mathrm{mag}$. Remarkably, one of these two stars has a large rotational velocity $\left(v \sin i=60 \pm 5 \mathrm{~km} \mathrm{~s}^{-1}\right.$; SO420039), while the another star falls in the halo of the $\sigma$ Orionis cluster
( $\rho \sim 23$ arcmin; SO211394), where the contamination by neighbouring young stellar populations becomes higher. An heliocentric distance of $d \sim 400 \mathrm{pc}$ is in agreement with recent results on the distance to the $\sigma$ Orionis cluster in Sherry et al. (2008) and Caballero (2008a). These authors claimed that distances of $d=420 \pm 30$ and $\sim 385 \mathrm{pc}$, respectively, are more plausible than previous estimates at $350 \mathrm{pc}$. However, our error bars are so large that we cannot favour a distance of $450 \mathrm{pc}$ rather than $350 \mathrm{pc}$.

\subsection{Lithium abundance}

Five stars in the selected sample have lithium abundances consistent within the error bars or slightly lower than the solar meteoritic value, $\log \epsilon(\mathrm{Li})=3.3$, which is also considered as the current Galactic or "cosmic" 7 $\mathrm{Li}$ abundance (Boesgaard \& Tripicco 1986). Therefore, young population I stars are believed to have inherited this lithium abundance at birth. However, two stars (SO131161 and SO210434) show higher lithium abundances, $\log \epsilon(\mathrm{Li}) \sim 3.6-3.7$ which might be due to wrong stellar parameters. Note that a $100 \mathrm{~K}$ cooler effective temperature would provide a $0.10-0.15$ dex lower lithium abundance. We should note that the uncertainties on the lithium abundances are quite high due to the large error bars of the temperature determination. Thus, some stars have lithium abundances that are at least marginally consistent, within the error bars, with the "cosmic" ${ }^{7} \mathrm{Li}$ abundance.

On the other hand, such high lithium abundances have been found in other T Tauri stars. For instance, Martín et al. (1994) showed two stars with similar stellar parameters $\left(T_{\mathrm{eff}} \sim 4700 \mathrm{~K}\right.$ and $\log g \sim 3.6-4.0)$ and LTE lithium abundances $\log \epsilon(\mathrm{Li}) \sim$ 3.65-3.60. However, their NLTE corrections were between -0.3 and -0.2 dex, thus significantly larger than our NLTE corrections, $\left|\Delta_{\text {NLTE-LTE }}\right| \lesssim-0.10$ dex. Those corrections moved their stars to the region of normal lithium abundances. In addition, a high NLTE lithium abundance has also been found in a doublelined spectroscopic binary of the $\sigma$ Orionis cluster by Lee et al. (1994): Mayrit 1415279 AB (discussed in Sect. 1). 


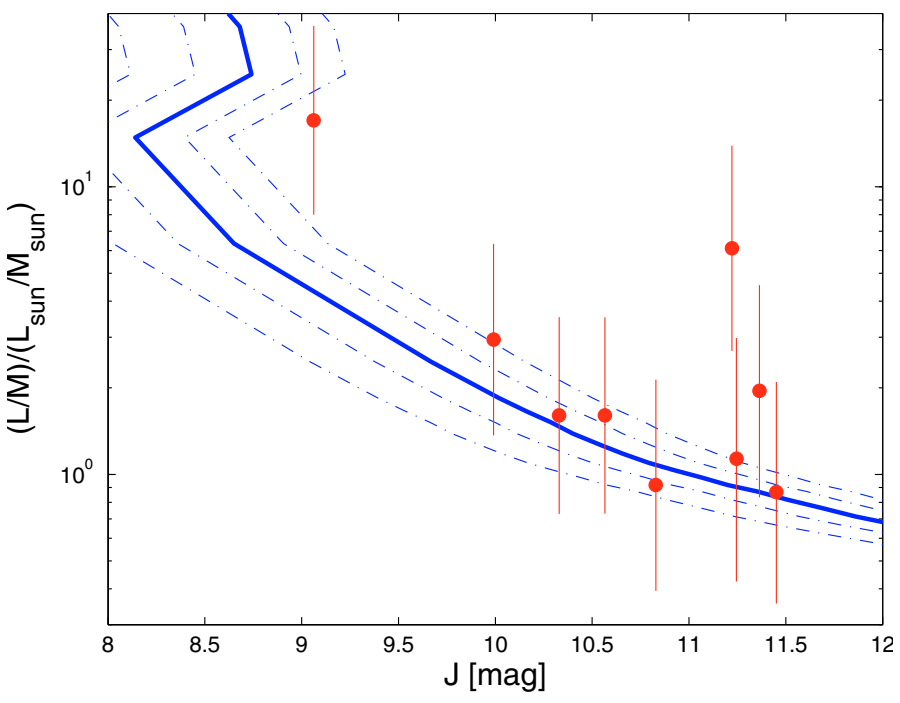

Fig. 3. Same as Fig. 2 but for the normalized luminosity per unit of mass, $\mathcal{L}$, as a function of the apparent magnitude, $J$. Dashed-dotted lines: tracks for fixed age ( $3 \mathrm{Ma})$, metallicity $(Z=0.02)$, and overshooting, and variable heliocentric distances $d=500,450,350,300 \mathrm{pc}$, from top to bottom; and thick solid line: track for $400 \mathrm{pc}, 3 \mathrm{Ma}, Z=0.02$, and overshooting.

Likewise, there are two additional stars with a lithium abundance slightly lower than the others, SO211394 $(\log \epsilon(\mathrm{Li})=$ $2.77 \pm 0.31)$ and SO000041 $(\log \epsilon(\mathrm{Li})=2.75 \pm 0.18)$. SO211394 is one of the three outliers in the $\mathcal{L}$ vs. $J$ diagram in Fig. 3 . The location of this star in the halo of the $\sigma$ Orionis cluster, its relatively low lithium abundance, and the abnormally faint $J$-band magnitude for its luminosity per unit of mass may suggest classifying the star as a member of a neighbouring, not-so-young population in the Ori OB1b association different to $\sigma$ Orionis (see, e.g., Jeffries et al. 2006). Given its position to the west of the cluster, SO211394 may belong to the extended population of the $\sim 5 \mathrm{Ma}$-old surrounding Alnilam (Collinder 70 or the " $\epsilon$ Orionis cluster"; Caballero \& Solano 2008). Nevertheless, its position in a $E W\left(\mathrm{Li}\right.$ I) vs. $T_{\text {eff }}$ diagram (e.g. López-Santiago et al. 2006) might also indicate that the star is a member of a distant $(d=710 \pm 50 \mathrm{pc})$ moving group with a Pleiades-like age.

SO000041 has a position only marginally consistent with the theoretical tracks in the $\mathcal{L}$ vs. $J$ diagram. This fact, together with the rather high proper motion of SO000041 (Sect. 1) and its location even further from the cluster centre than SO211394, casts doubts on its membership of the $\sigma$ Orionis cluster. SO000041 is the easternmost, bright cluster member candidate in the Mayrit catalogue, just in the outskirts of the Horsehead Nebula (Caballero 2007, 2008c). Membership in a slightly younger population of recently born stars associated with the nebula might explain its relatively low gravity. However, membership of a $\sim 100$ Ma-old moving group (like SO211394) at $d \sim 630 \pm 50 \mathrm{pc}$ could explain both its relatively low lithium abundance and large proper motion. Further astrometric and spectrophotometric studies are needed to ascertain the real nature of SO000041 and SO211394.

\subsection{Metallicity of $\sigma$ Orionis}

The nine selected stars share the same radial velocity and have high $\mathrm{Li}$ abundances consistent with the "cosmic" ${ }^{7} \mathrm{Li}$ abundance, so we think that they are probably members of the $\sigma$ Orionis cluster. According to the values shown in Table 3, the average metallicity of these nine selected stars (i.e. the metallicity of the $\sigma$ Orionis cluster) is $[\mathrm{Fe} / \mathrm{H}]=-0.02 \pm 0.09$ (random) \pm 0.13 (systematic). This value, as well as those for other element abundances, is consistent with solar metallicity. If we do not take into account SO131161 and SO230062, that are not Mayrit objects (the two no Mayrit stars are at angular separations $\rho>30$ arcmin to the $\sigma$ Orionis centre, and might not belong to the cluster - Jeffries et al. 2006; Caballero 2007, 2008b), the new average metallicity barely varies from $[\mathrm{Fe} / \mathrm{H}]=-0.05 \pm 0.08 \pm 0.13$. Both metallicities would provide a distance to the cluster of $d \sim 440$ pc from the results presented by Sherry et al. (2008). If we also remove from the sample the star SO420039 (that seems to be at a greater heliocentric distance; Sect. 4.1) and the stars SO211394 and SO000041 (that have relatively low lithium abundances; Sect. 4.2), then the average metallicity is $[\mathrm{Fe} / \mathrm{H}]=0.00 \pm 0.07 \pm 0.13$.

\section{Summary}

We have presented WHT/WYFFOS+AF2 spectroscopy of a sample of 24 young late-type stars of the $\sigma$ Orionis cluster, for which we have compiled optical and near infrared photometry, radial and rotational velocities, and effective temperatures derived with the infrared flux method. Among this sample, we have selected nine late-type single stars with high enough quality spectra and effective temperatures in a suitable interval for an accurate abundance analysis. We have applied a $\chi^{2}$-minimization technique that compares a grid a synthetic spectra with the observations. This method provides a determination of the stellar parameters and metallicity.

Including the nine stars in the selected sample, the average metallicity for the $\sigma$ Orionis cluster is $[\mathrm{Fe} / \mathrm{H}]=-0.02 \pm$ $0.09 \pm 0.13$ (random and systematic errors), and thus is in close agreement with solar metallicity. The element abundances of aluminium, silicon, calcium, and nickel also show solar values. We have determined the lithium abundances of the nine selected stars. Except for two stars (SO230062 and SO210434), the lithium abundances are similar to or slighty lower than the "cosmic" ${ }^{7} \mathrm{Li}$ abundance, within the error bars. These two stars show a lithium abundance of $\log \epsilon(\mathrm{Li})=3.6-3.7$, although almost consistent with the "cosmic" ${ }^{7} \mathrm{Li}$ abundance within the error bars.

The stellar parameters of the remaining selected stars are consistent with a heliocentric distance in the range $350<d / \mathrm{pc}<$ 500 and an age of $3 \pm 1 \mathrm{Ma}$ for the $\sigma$ Orionis cluster. Finally, we have determined the mean radial velocity of the cluster at $V_{\mathrm{r}}=+28 \pm 4 \mathrm{~km} \mathrm{~s}^{-1}$.

Thus, the metallicity of the $\sigma$ Orionis cluster is solar within the uncertainties of our method. Most of the investigated stars also display cosmic lithium abundances, consistent with their expected very young ages, although at least two of them would deserve dedicated spectroscopic follow-up at higher resolution to confirm or reject their membership of $\sigma$ Orionis.

Acknowledgements. We are grateful to Tom Marsh for the use of the MOLLY analysis package. We thank the supporter astronomer, R. Corradi, during our observations at the WHT. This work has made use of the VALD database and IRAF facilities. J.I. ackonowledges support from the EU contract MEXTCT-2004-014265 (CIFIST). Partial financial support was provided by Spanish Ministerio Educación y Ciencia, the Universidad Complutense de Madrid, the Spanish Virtual Observatory under grants AyA2005-05149, AyA2005-02750, AyA2005-04286 and AyA2005-24102-E of the Programa Nacional de Astronomía y Astrofísica and by the Comunidad Autónoma de Madrid under PRICIT project S-0505/ESP-0237 (AstroCAM), MEC/Consolider-CSD20060070 and CAM/PRICIT-S-0505/ESP/0361. 


\section{References}

Alcalá, J. M., Covino, E., Torres, G., et al. 2000, A\&A, 353, 186

Allende Prieto, C., Barklem, P. S., Lambert, D. L., \& Cunha, K. 2004, A\&A, 53, 181

Al-Naimiy, H. M. 1978, Ap\&SS, 420, 183

Alonso, A., Arribas, S., \& Martínez-Roger, C. 1995, A\&A, 297, 197

Alonso, A., Arribas, S., \& Martínez-Roger, C. 1999, A\&AS, 139, 335

Barrado y Navascués, D., Zapatero Osorio, M. R., Béjar, V. J. S., et al. 2001, A\&A, 377, L9

Barrado y Navascués, D., Zapatero Osorio, M. R., Martín, E. L., et al. 2002, A\&A, 393, L85

Béjar, V. J. S., Zapatero Osorio, M. R., \& Rebolo, R. 1999, ApJ, 521, 671

Béjar, V. J. S., Zapatero Osorio, M. R., \& Rebolo, R. 2004, AN, 325, 705

Bensby, T., Feltzing, S., Lundström, I., \& Ilyin, I. 2005, A\&A, 433, 185

Bessell, M. S., \& Brett, J. M. 1988, PASP, 100, 1134

Boesgaard, A. M., \& Tripicco, M. J. 1986, ApJ, 303, 724

Boss, A. P. 1997, Science, 276, 1836

Brown, A. G. A., de Geus, E. J., \& de Zeeuw, P. T. 1994, A\&A, 289, 101

Burningham, B., Naylor, T., Littlefair, S. P., \& Jeffries, R. D. 2005, MNRAS 356,1583

Caballero, J. A. 2005, AN, 326, 1007

Caballero, J. A. 2006, Ph.D. Thesis, Universidad de La Laguna, Tenerife, Spain

Caballero, J. A. 2007, A\&A, 466, 917

Caballero, J. A. 2008a, MNRAS, 383, 375

Caballero, J. A. 2008b, MNRAS, 383, 750

Caballero, J. A. 2008c, A\&A, 478, 667

Caballero, J. A., \& Solano, E. 2008, A\&A, 485, 931

Caballero, J. A., Martín, E. L., Dobbie, P. D., \& Barrado y Navascués, D. 2006 A\&A, 460, 635

Caballero, J. A., Béjar, V. J. S., Rebolo, R., et al. 2007, A\&A, 470, 903

Cohen, M., Wheaton, W. A., \& Megeath, S. T. 2003, AJ, 126, 1090

Cunha, K., \& Lambert, D. L. 1994, ApJ, 426, 170

Cunha, K., Smith, V. V., \& Lambert, D. L. 1998, ApJ, 493, 195

Cunha, K., Smith, V. V., Parizot, E., \& Lambert, D. L. 2000, ApJ, 543, 850

Epchtein, N., de Batz, B., Capoani, L., et al. 1997, Msngr, 87, 27

Fischer, D. A., \& Valenti, J. 2005, ApJ, 662, 1102

Franciosini, E., Pallavicini, R., \& Sanz-Forcada, J. 2006, A\&A, 446, 50

González Hernández, J. I., Rebolo, R., Israelian, G., et al. 2004, ApJ, 609, 988

González Hernández, J. I., Rebolo, R., Israelian, G., et al. 2005, ApJ, 630, 495

González Hernández, J. I., Rebolo, R., \& Israelian, G. 2008, A\&A, 478, 203

Grevesse, N., Noels, A., \& Sauval, A. J. 1996, Proceedings of the sixth annua October Astrophysics Conference in College Park, Maryland, 9-11 October 1995 (San Francisco: ASP), ed. S. S. Holt, \& G. Sonneborn, ASP Conf. Ser., 99, 117

Gregorio-Hetem, J., \& Hetem, A. 2002, MNRAS, 336, 197

Ida, S., \& Lin, D. N. C. 2004, ApJ, 662, 1067

Jeffries, R. D., Maxted, P. F. L., Oliveira, J. M., \& Naylor, T. 2006, MNRAS, 371, L6

Kenyon, M. J., Jeffries, R. D., Naylor, T., Oliveira, J. M., \& Maxted, P. F. L. 2005, MNRAS, 356, 89

Kurucz, R. L. 1993, ATLAS9 Stellar Atmospheres Programs and $2 \mathrm{~km} \mathrm{~s}^{-1}$ Grid, CD-ROM No. 13, Smithsonian Astrophysical Observatory (Cambridge, MA USA)
Kurucz, R. L., Furenild, I., Brault, J. \& Testerman, L. 1984, Solar Flux Atlas from 296 to $1300 \mathrm{~nm}$, National Solar Observatory Atlas, Sunspot, New Mexico (Cambridge: Harvard Univ. Press)

Lee, T. A. 1968, ApJ, 152, 913

Lee, C. W., Martín, E. L., \& Mathieu, R. D. 1994, AJ, 108, 1445

Lissauer, J. J. 1993, ARA\&A, 31, 129

López-Santiago, J., \& Caballero, J. A. 2008, A\&A, in press [arXiv:0809.0990]

López-Santiago, J., Montes, D., Crespo-Chacón, I., \& Fernández-Figueroa, M. J. 2006, ApJ, 643, 1160

Luhman, K. L., Hernández, J., Downes, J. J., Hartmann, L., \& Briceño, C. 2008, ApJ, in press [arXiv: 0808.0471$]$

Martín, E. L., \& Zapatero Osorio, M. R. 2003, ApJ, 593, L113

Martín, E. L., Rebolo, R., Magazzù, A., \& Pavlenko, Ya. V. 1994, A\&A, 282, 503

Martín, E. L., Zapatero Osorio, M. R., Barrado y Navascués, D., Béjar, V. J. S., \& Rebolo, R. 2001, ApJ, 558, L117

Maxted, P. F. L., Jeffries, R. D., Oliveira, J. M., Naylor, T., \& Jackson, R. J. 2008, MNRAS, 385, 2210

McWilliam, A. 1997, ARA\&A, 35, 503

Morrell, N., \& Levato, H. 1991, ApJS, 75, 965

Muzerolle, J., Hillenbrand, L., Calvet, N., Briceño, C., \& Hartmann, L. 2003, ApJ, 592, 266

Oliveira, J. M., Jeffries, R. D., Kenyon, M. J., Thompson, S. A., \& Naylor, T. 2002, A\&A, 382, L22

Pavlenko, Ya. V., \& Magazzù, A. 1996, A\&A, 311, 961

Piskunov, N. E., Kupka, F., Ryabchikova, T. A., Weiss, W. W., \& Jeffery, C. S. 1995, A\&AS, 112, 525

Pojmanski, G. 2002, AcA, 52, 397

Pollack, J. B., Hubickyj, O., Bodenheimer, P., et al. 1996, Icarus, 124, 62

Sacco, G. G., Randich, S., Franciosini, E., Pallavicini, R., \& Palla, F. 2008, A\&A, 488, 167

Sanz-Forcada, J., Franciosini, E., \& Pallavicini, R. 2004, A\&A, 421, 715

Schlegel, D. J., Finkbeiner, D. P., \& Davis, M. 1998, ApJ, 500, 525

Sneden, C. 1973, Ph.D. Thesis, University of Texas at Austin, TX, USA

Sherry, W. H., Walter, F. M., \& Wolk, S. J. 2004, AJ, 128, 2316

Scholz, A., \& Jayawardhana, R. 2008, ApJ, 672, L49

Sherry, W. H., Walter, F. M., Wolk, S. J., \& Adams, N. R. 2008, AJ, 135, 1616

Siess, L., Dufour, E., \& Forestini, M. 2000, A\&A, 358, 593

Skinner, S. L., Sokal, K. R., Cohen, D. H., et al. 2008, ApJ, 683, 796

Skrutskie, M. F., Cutri, R. M., Stiening, R., et al. 2006, AJ, 131, 1163

Wilson, R. E. 1953, Carnegie Institute Washington D.C. Publication, 601, 1953 $(\mathrm{XXBH})$

Wolk, S. J. 1996, Ph.D. Thesis, State University New York at Stony Brook, NY, USA

Zapatero Osorio, M. R., Béjar, V. J. S., Martín, E. L., et al. 2000, Science, 290, 103

Zapatero Osorio, M. R., Béjar, V. J. S., Pavlenko, Ya., et al. 2002a, A\&A, 384, 937

Zapatero Osorio, M. R., Béjar, V. J. S., Martín, E. L., et al. 2002b, ApJ, 569, L99 Zapatero Osorio, M. R., Béjar, V. J. S., Martín, E. L., et al. 2002c, ApJ, 578, 536 Zapatero Osorio, M. R., Caballero, J. A., Béjar, V. J. S., et al. 2007, A\&A, 472, L9 